\title{
On the Structure of Correction Matrices for a $k$-Exact High-Order Finite-Volume Scheme on Vertex-Centered Unstructured Grids
}

\author{
Florian Setzwein* and Peter Ess ${ }^{\dagger}$ \\ Institute of Combustion Technology, German Aerospace Center (DLR), Stuttgart, 70569, \\ Germany \\ Moritz Spraul ${ }^{\ddagger}$ and Peter Gerlinger ${ }^{\S}$ \\ IVLR, University of Stuttgart, Stuttgart, 70569, Germany
}

\begin{abstract}
The $k$-exact multiple correction scheme is a promising approach to incorporate a highorder accuracy into existing unstructured Finite-Volume solvers. Essential for this scheme are recursive applications of correction matrices on Green-Gauss derivatives, which enhance the accuracy and require only the exchange of information between adjacent elements. In this work, a detailed analysis of the eigenstructure of these correction matrices is employed for a vertex-centered multiple-correction scheme, that features spatially a third order of accuracy on unstructured meshes. It is presented, how the matrix eigenvalues change due to deformation for a single element. Furthermore, we demonstrate that these eigenvalues can be used for the calculation of a new grid metric, which is an excellent indicator for the distortion and the connectivity of a grid. The relation of this indicator to the numerical error is highlighted through a linear advection benchmark for various discretization schemes. Through this it is also shown, that the $k$-exact multiple-correction schemes are less affected by grid distortions than conventional schemes.
\end{abstract}

\section{Introduction}

Due to enhanced resolution properties, higher-order methods can significantly reduce the computational effort for the simulation of complex flows in comparison to conventional discretization schemes $!^{1}$ In recent years, more and more methods have been established which enable a higher order of accuracy to be realized even for complex geometries. Examples include the Discontinuous Galerkin and Spectral Volume methods or the Flux Reconstruction approach. ${ }^{[2}$ However, a considerable effort is required to implement such high-order methods into existing flow solvers, since these are often based on the Finite-Volume method and are therefore limited due to the underlying data structure. The $k$-exact reconstruction approach offers the possibility to increase the spatial accuracy of Finite-Volume solvers with moderate effort and can significantly improve the accuracy of the solution. The approach is based on a generalized Godunov scheme and goes back to the work of Barth and Frederickson $3 \mid 4$ The solution is reconstructed locally in every control volume by means of polynomial functions of degree $k$, whose coefficients are generally estimated through a least-squares approach from volume-averages of a compact stencil of elements. However, as the order of accuracy increases, these stencils must include a higher number of elements to maintain a robust discretization scheme. This leads to parallelization concerns since information is required from elements that are not adjacent. Haider et al ${ }^{[57}$ presented a general procedure to maintain $k$-exactness on unstructured grids, that only requires the

*PhD Student, German Aerospace Center (DLR), Stuttgart, 70569, Germany

${ }^{\dagger}$ Postdoctoral Fellow, Senior AIAA Member, German Aerospace Center (DLR), Stuttgart, 70569, Germany

${ }^{\ddagger}$ Student, IVLR University of Stuttgart, 70569, Germany

$\S$ Professor, IVLR University of Stuttgart, Stuttgart, 70569, Germany 
exchange of information between face-neighboring elements and thus enhances the parallelization of the scheme. It is based on a recursive correction of approximate successive derivatives, which are estimated with a least-squares approach. The approach was adapted in the so called multiple-correction approach by Pont et al ${ }^{8}$ Here, the required derivatives are calculated with a Green-Gauss formulation, which results in a better performance on highly deformed grids. The correction of the Green-Gauss gradients is based on correction matrices, which ensure the $k$-exactness of derivatives even on highly distorted grids. The approach, initially derived for cell-centered grids, has recently been extended for vertex-centered grids by Setzwein et al ${ }^{9}$ Vertex-centered grids typically feature a higher number of neighbors per element and thus enhance the overall approximation, since more degrees of freedom are available loacally to estimate the reconstruction polynomials. The major difference to the cell-centered approach is the calculation of the correction matrices. This results from the fact, that the flow data in the vertex-centered approach is generally not stored at the elements geometric centroids. ${ }^{9}$ This difference is incorporated into the correction matrices through grid dependent metrics, that are referred to as geometric volume moment tensors. Since these correction matrices contain so much information about the grid structure, it should be possible to exploit them to measure the grid quality. Furthermore, a better understanding on how these matrices behave upon mesh deformation will lead to a further insight into the $k$-exact multiple-correction approach for vertex-centered grids.

\section{The $k$-Exact Multiple-Correction Approach}

This section gives a brief overview on the 2-exact reconstruction for vertex-centered unstructured grids. Details on the derivation of the scheme can be found in the authors previous work 9 The main purpose for the discretization scheme is to enhance the accuracy of the convective operator of any transport equation. We thus restrict ourselves to the discretization of the divergence form of the convective operator, which describes the convection of a physical quantity $\phi(\mathbf{x}, t)$ in an incompressible flowfield with velocity $\mathbf{u}(\mathbf{x}, t)$

$$
\frac{\partial \phi}{\partial t}+\nabla \cdot(\mathbf{u} \phi)=0
$$

Equation (1) is solved in a domain $\Omega \subset \mathbb{R}^{d}$, which is discretized by a mesh of $N$ non-overlapping complex polyhedral cells. This mesh is referred to as the median dual grid tesselation $\mathcal{D}(\Omega)$ and it is the edge-based representation of a primary grid $\mathcal{P}(\Omega)$ consisting of linear elements. Two elements $\Omega_{\alpha}$ and $\Omega_{\beta}$ are considered to be adjacent if they share a commong face $A_{\alpha \beta}$. All adjacent elements of a cell $\Omega_{\alpha}$ are referred to as its $1^{\text {st }}$ neighborhood, signed as $\mathbb{V}_{\alpha}^{(1)}$. The $n^{\text {th }}$ neighborhood of $\Omega_{\alpha}$ is defined recursively via the neighborhoods of its adjacent elements $\mathbb{V}_{\alpha}^{(n)}:=\bigcup_{\gamma \in \mathbb{V}_{\alpha}^{(n-1)}} \mathbb{V}_{\gamma}^{(1)}$. Figure 1 shows a primary grid $\mathcal{P}(\Omega)$, its respective median dual tesselation $\mathcal{D}(\Omega)$ and the first and second neighborhood of a median dual cell $\Omega_{\alpha}$ for $d=2$.

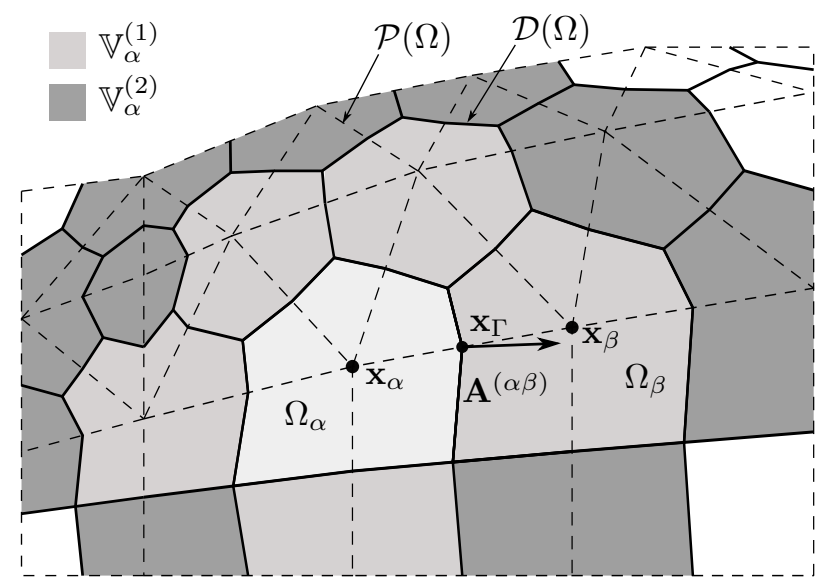

Figure 1: Median dual grid in 2D, indicated in solid lines. The corresponding primary grid is drawn in dashed lines. The simulation variables are stored at the location of primary grid nodes, e.g. $\mathbf{x}_{\alpha}$ or $\mathbf{x}_{\beta}$. 
Fundamental for the discretization scheme is the volume-averaging operator, which is defined for any element $\Omega_{\alpha}$ through

$$
\bar{\phi}_{\alpha}:=\frac{1}{\left|\Omega_{\alpha}\right|} \iiint_{\Omega_{\alpha}} \phi(\mathbf{x}) \mathrm{d} V .
$$

In the scope of a Godunov scheme, the volume-averaging is applied to equation (1) and the volume-averages $\bar{\phi}$ act as degrees of freedom, which leads to a system of $N$ equations:

$$
\frac{\partial \bar{\phi}_{\alpha}}{\partial t}+\frac{1}{\left|\Omega_{\alpha}\right|} \sum_{\beta \in \mathbb{V}_{\alpha}^{(1)}} \iint_{A_{\alpha \beta}}(\mathbf{u} \phi) \cdot \mathbf{n} \mathrm{d} A=0 .
$$

The surface integrals are referred to as fluxes and must be approximated in order to close the system of equations. Essentially, three steps are required to solve equation (3) with the $k$-exact reconstruction method ${ }^{3}$ First, the solution for every primitive field variable is reconstructed locally in the vicinity of each element $\Omega_{\alpha}$ from the known volume-averages at a timestep $t_{n}$. The fluxes at the element interfaces are then approximated with a higher order accuracy using the reconstructed solution of the adjacent elements. Finally, the system of equations is evolved in time by solving for new volume-averages at timestep $t_{n+1}$. In this work, a 2-exact the multiple-correction approach is employed for the reconstruction step and Taylor-polynomials are used to approximate the solution in the vicinity of an element $\Omega_{\alpha}$. This leads to the following definition for the 2-exact reconstruction polynomial of the scalar quantity $\phi$ :

$$
\phi_{\alpha}^{(2)}(\mathbf{x})=\phi_{\alpha}+\frac{\partial}{\partial x_{i}}[\bar{\phi}]_{\alpha}^{* *}\left(x_{i}-x_{i, \alpha}\right)+\frac{1}{2} \frac{\partial^{2}}{\partial x_{i} \partial x_{j}}[\bar{\phi}]_{\alpha}^{*}\left(x_{i}-x_{i, \alpha}\right)\left(x_{j}-x_{j, \alpha}\right)+\mathcal{O}\left(h^{3}\right),
$$

with $h$ being a characteristic cell width of the median dual mesh. The value $\phi_{\alpha}$ refers to a 2-exact approximation of $\phi$ at the primary grid node $\mathbf{x}_{\alpha}$. It can be estimated if the volume-averaging operation is applied to the reconstruction polynomial (4)

$$
\phi_{\alpha}=\bar{\phi}_{\alpha}-\frac{\partial}{\partial x_{i}}[\bar{\phi}]_{\alpha}^{* *} \mathcal{M}_{i}^{(\alpha, \alpha)}-\frac{1}{2} \frac{\partial^{2}}{\partial x_{i} \partial x_{j}}[\bar{\phi}]_{\alpha}^{*} \mathcal{M}_{i j}^{(\alpha, \alpha)}+\mathcal{O}\left(h^{3}\right) .
$$

The terms $\mathcal{M}_{i}^{(\alpha, \alpha)}$ and $\mathcal{M}_{i j}^{(\alpha, \alpha)}$ refer to as rank $p$ geometric volume moment tensors and can be calculated analytically prior to the simulation according to

$$
\mathcal{M}_{i_{1} i_{2} \ldots i_{p}}^{(\beta, \alpha)}=\frac{1}{\left|\Omega_{\beta}\right|} \iiint_{\Omega_{\beta}}\left(x_{i_{1}}-x_{i_{1}, \alpha}\right)\left(x_{i_{2}}-x_{i_{2}, \alpha}\right) \ldots\left(x_{i_{p}}-x_{i_{p}, \alpha}\right) \mathrm{d} V .
$$

The first superscript $\beta$ denotes the volume $\Omega_{\beta}$ for performing the averaging and the second superscript $\alpha$ denotes the point $\mathbf{x}_{\alpha}$ used for centering the moment. Only moment tensors $\mathcal{M}_{i_{1} i_{2} \ldots i_{p}}^{(\alpha, \alpha)}$ must be stored for each element, since the moment tensor $\mathcal{M}_{i_{1} i_{2} \ldots i_{p}}^{(\beta, \alpha)}$ between two adjacent cells $\alpha$ and $\beta$ can be deduced from binomial expansion $\frac{10}{11}$ Finally, in order to maintain a third order accurate reconstruction of $\phi$ on $\Omega_{\alpha}$, it remains to approximate the unknown derivative operators $\frac{\partial}{\partial x_{i}}[\bar{\phi}]_{\alpha}^{* *}$ with an accuracy of $\mathcal{O}\left(h^{2}\right)$ and $\frac{\partial^{2}}{\partial x_{i} \partial x_{j}}[\bar{\phi}]_{\alpha}^{*}$ with $\mathcal{O}(h)$. This could be done via a least-squares approach over a stencil of elements in the vicinity of $\Omega_{\alpha}$. But especially for $d=3$ a high number of elements is required to ensure a stable reconstruction. This would lead to performance losses and would complicate the parallelization of the scheme, since many elements must be accessed that are not adjacent to another. This problem does not arise for the multiple-correction method, because the unknown derivatives are determined through approximate Green-Gauss gradients, that only involve exchange of information between adjacent elements. Since these derivatives do not meet the $k$ exactness criterion, they must be successively corrected to higher levels of accuracy using geometric matrices, which are calculated prior to the simulation. The first derivative to compute is a 0 -exact gradient operator, which yields an error of $\mathcal{O}(1)$ on deformed meshes:

$$
\frac{\partial}{\partial x_{j}}[\bar{\phi}]_{\alpha}:=\frac{1}{\left|\Omega_{\alpha}\right|} \sum_{\beta \in \mathbb{V}_{\alpha}^{(1)}} \frac{1}{2}\left(\bar{\phi}_{\beta}+\bar{\phi}_{\alpha}\right) \mathcal{S}_{j}^{(\alpha \beta)} .
$$

This gradient is multiplied with a matrix $\mathbf{G}_{\alpha}$ in order to obtain a 1-exact gradient formulation that ensures an accuracy of $\mathcal{O}(h)$ on arbitrary grids. The resulting 1-exact gradient operator at the cell centroid $\mathbf{x}_{\alpha}$ is 
indicated with an asterisk

$$
\frac{\partial}{\partial x_{i}}[\bar{\phi}]_{\alpha}^{*}:=G_{i j, \alpha} \frac{\partial}{\partial x_{j}}[\bar{\phi}]_{\alpha} .
$$

It can be shown, that the inverse of the gradient correction matrix $\mathbf{G}_{\alpha}$ must be calculated for vertex-centered grids through

$$
G_{i j, \alpha}^{-1}=\frac{\partial}{\partial x_{i}}\left[\mathcal{M}_{j}\right]_{\alpha} .
$$

The entries of this matrix are related to the 0 -exact gradient of the first geometric volume moments of $\Omega_{\alpha}$

$$
\frac{\partial}{\partial x_{i}}\left[\mathcal{M}_{j}\right]_{\alpha}:=\frac{1}{\left|\Omega_{\alpha}\right|} \sum_{\beta \in \mathbb{V}_{\alpha}^{(1)}} \frac{1}{2}\left(\mathcal{M}_{j}^{(\beta, \alpha)}+\mathcal{M}_{j}^{(\alpha, \alpha)}\right) \mathcal{S}_{i}^{(\alpha \beta)} .
$$

This expression is simply a Green-Gauss gradient operation applied to the respective geometric moment tensor entries. It must be noticed that the centering of the geometric volume moments in equation (10) varies according to the respective element $\Omega_{\alpha}$. Figure 2 shows the influence of the deformation of a median dual element $\Omega_{\alpha}$ on its surrounding elements. The deformation leads to a change of the geometric volume moments $\mathcal{M}_{j}$ of $\Omega_{\alpha}$ and its neighbors. This change is incorporated in the matrix $\mathbf{G}_{\alpha}$ and is used to correct the approximate Green-Gauss gradients. Therefore, the matrix should contain information about the distorition of a grid, which could be used to inidcate the mesh quality prior to the simulation. This will be examined in detail within this paper using the matrix eigenvalues.

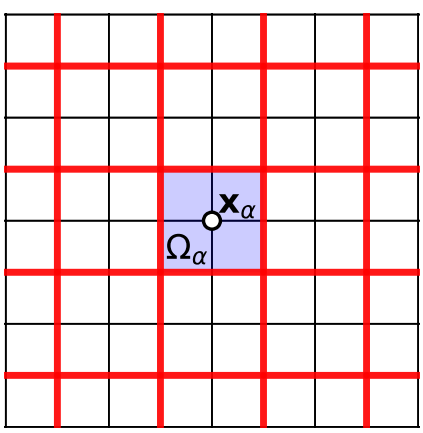

(a) No deformation

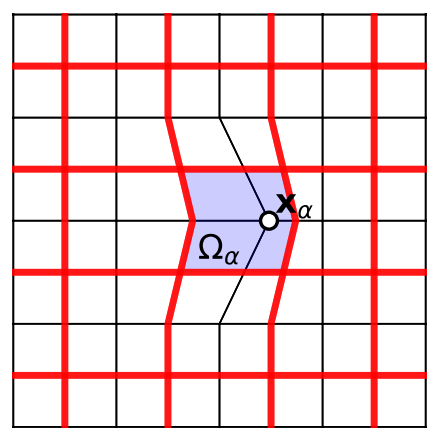

(b) Deformation in $x_{1}$-direction

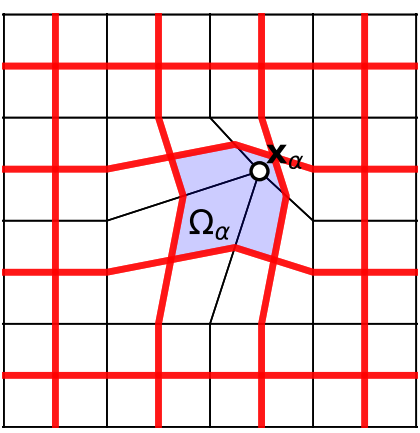

(c) Deformation in $x_{1}-x_{2}$-direction

Figure 2: Influence of the deformation of $\Omega_{\alpha}$ (indicated in blue) on its surrounding elements. Red thick lines denote the median dual grid and black thin lines indicate the primary grid.

The second derivative of $\phi(\mathbf{x})$ is approximated by applying the Green-Gauss gradient operator on the 1 -exact gradient of the field variable $\phi$. This leads to the approximated Hessian matrix operator $\frac{\partial^{2}}{\partial x_{i} \partial x_{j}}[\phi]_{\alpha}$ with an accuracy of $\mathcal{O}(1)$ on deformed meshes

$$
\frac{\partial^{2}}{\partial x_{i} \partial x_{j}}[\bar{\phi}]_{\alpha}:=\frac{\partial}{\partial x_{i}}\left[\frac{\partial}{\partial x_{j}}[\bar{\phi}]^{*}\right]_{\alpha}=\frac{1}{\left|\Omega_{\alpha}\right|} \sum_{\beta \in \mathbb{V}_{\alpha}^{(1)}} \frac{1}{2}\left(\frac{\partial}{\partial x_{j}}[\phi]_{\beta}^{*}+\frac{\partial}{\partial x_{j}}[\phi]_{\alpha}^{*}\right) \mathcal{S}_{i}^{(\alpha \beta)} .
$$

Similar to the 0 -exact gradient, there exists a linear mapping, which can be used to correct $\frac{\partial^{2}}{\partial x_{i} \partial x_{j}}[\bar{\phi}]_{\alpha}$ onto a form that ensures an accuracy of $\mathcal{O}(h)$ on arbitrary grids. To express this linear mapping in terms of a matrix multiplication, all symmetric rank two tensors are vectorized, as for example the Hessian matrix and the geometric volume tensor. Such vectorized tensors will be denoted by a circumflex and their entries are all arranged in the same order. For example, the vectorized 0-exact Hessian matrix $\frac{\partial^{2}}{\partial x_{i} \partial x_{j}}[\bar{\phi}]_{\alpha}$ reads

$$
\widehat{\mathbf{D}}^{(2)}[\bar{\phi}]_{\alpha}=\left(\frac{\partial^{2}}{\partial x_{1} \partial x_{1}}[\bar{\phi}]_{\alpha} \frac{\partial^{2}}{\partial x_{1} \partial x_{2}}[\bar{\phi}]_{\alpha} \frac{\partial^{2}}{\partial x_{1} \partial x_{3}}[\bar{\phi}]_{\alpha} \frac{\partial^{2}}{\partial x_{2} \partial x_{2}}[\bar{\phi}]_{\alpha} \frac{\partial^{2}}{\partial x_{2} \partial x_{3}}[\bar{\phi}]_{\alpha} \frac{\partial^{2}}{\partial x_{3} \partial x_{3}}[\bar{\phi}]_{\alpha}\right)^{T} \text {. }
$$


Similar to the 1-exact gradient operator, the 0-exact Hessian matrix operator is corrected with a matrix $H_{i j, \alpha}$

$$
\widehat{D}_{i}^{(2)}[\bar{\phi}]_{\alpha}^{*}:=H_{i j, \alpha} \widehat{D}_{j}^{(2)}[\bar{\phi}]_{\alpha} .
$$

The inverse of this Hessian correction matrix $\mathbf{H}_{\alpha}$ is defined through

$$
\mathbf{H}_{\alpha}^{-1}=\left[\begin{array}{ccccc}
1+\frac{\partial^{2}}{\partial x_{1} \partial x_{1}}\left[\mathcal{M}_{11}\right]_{\alpha} & 2 \frac{\partial^{2}}{\partial x_{1} \partial x_{1}}\left[\mathcal{M}_{12}\right]_{\alpha} & 2 \frac{\partial^{2}}{\partial x_{1} \partial x_{1}}\left[\mathcal{M}_{13}\right]_{\alpha} & \ldots & \frac{\partial^{2}}{\partial x_{1} \partial x_{1}}\left[\mathcal{M}_{33}\right]_{\alpha} \\
\frac{\partial^{2}}{\partial x_{1} \partial x_{2}}\left[\mathcal{M}_{11}\right]_{\alpha} & 1+2 \frac{\partial^{2}}{\partial x_{1} \partial x_{2}}\left[\mathcal{M}_{12}\right]_{\alpha} & 2 \frac{\partial^{2}}{\partial x_{1} \partial x_{2}}\left[\mathcal{M}_{13}\right]_{\alpha} & \ldots & \frac{\partial^{2}}{\partial x_{1} \partial x_{2}}\left[\mathcal{M}_{33}\right]_{\alpha} \\
\vdots & \vdots & \vdots & \ldots & \vdots \\
\frac{\partial^{2}}{\partial x_{3} \partial x_{3}}\left[\mathcal{M}_{11}\right]_{\alpha} & 2 \frac{\partial^{2}}{\partial x_{3} \partial x_{3}}\left[\mathcal{M}_{12}\right]_{\alpha} & 2 \frac{\partial^{2}}{\partial x_{3} \partial x_{3}}\left[\mathcal{M}_{13}\right]_{\alpha} & \ldots & 1+\frac{\partial^{2}}{\partial x_{3} \partial x_{3}}\left[\mathcal{M}_{33}\right]_{\alpha}
\end{array}\right] .
$$

The expression $\frac{\partial^{2}}{\partial x_{i} \partial x_{j}}\left[\mathcal{M}_{k l}\right]_{\alpha}$ denotes the 0 -exact Hessian matrix operator of equation (11) applied on the rank two volume moment tensor. Since both $\mathbf{H}_{\alpha}$ and $\mathbf{G}_{\alpha}$ depend on approximate derivatives of the geometric volume moment tensors, they rely only on the mesh geometry and can be calculated and inverted prior to the simulation. Finally, a 2-exact gradient operator is estimated through a further correction operation. It introduces an error of $\mathcal{O}\left(h^{2}\right)$ and is defined according to

$$
\frac{\partial}{\partial x_{i}}[\bar{\phi}]_{\alpha}^{* *}:=\frac{\partial}{\partial x_{i}}[\bar{\phi}]_{\alpha}^{*}-\frac{1}{2} G_{i j, \alpha}^{*} \widehat{D}_{j}^{(2)}[\bar{\phi}]_{\alpha}^{*} .
$$

The $(3 \times 6)$ matrix $G_{i j, \alpha}^{*}$ is calculated according to

$$
\mathbf{G}_{\alpha}^{*}=\left[\begin{array}{ccccc}
\frac{\partial}{\partial x_{1}}\left[\mathcal{M}_{11}\right]_{\alpha}^{*} & 2 \frac{\partial}{\partial x_{1}}\left[\mathcal{M}_{12}\right]_{\alpha}^{*} & 2 \frac{\partial}{\partial x_{1}}\left[\mathcal{M}_{13}\right]_{\alpha}^{*} & \cdots & \frac{\partial}{\partial x_{1}}\left[\mathcal{M}_{33}\right]_{\alpha}^{*} \\
\frac{\partial}{\partial x_{2}}\left[\mathcal{M}_{11}\right]_{\alpha}^{*} & 2 \frac{\partial}{\partial x_{2}}\left[\mathcal{M}_{12}\right]_{\alpha}^{*} & 2 \frac{\partial}{\partial x_{2}}\left[\mathcal{M}_{13}\right]_{\alpha}^{*} & \cdots & \frac{\partial}{\partial x_{2}}\left[\mathcal{M}_{33}\right]_{\alpha}^{*} \\
\frac{\partial}{\partial x_{3}}\left[\mathcal{M}_{11}\right]_{\alpha}^{*} & 2 \frac{\partial}{\partial x_{3}}\left[\mathcal{M}_{12}\right]_{\alpha}^{*} & 2 \frac{\partial}{\partial x_{3}}\left[\mathcal{M}_{13}\right]_{\alpha}^{*} & \cdots & \frac{\partial}{\partial x_{3}}\left[\mathcal{M}_{33}\right]_{\alpha}^{*}
\end{array}\right]
$$

As soon as the derivatives for every field variable have been estimated, the flux function can be evaluated on the element interfaces. The flux integration is performed through a single point formula, which preserves a 2-exact reconstruction. The approach can be derived by expanding a Taylor-series of degree two around a single point $\mathbf{x}_{\Gamma}$ on the interface $A_{\alpha \beta}$ between two adjacent elements $\Omega_{\alpha}$ and $\Omega_{\beta}$. If all primitive field variables $\phi$ and $u_{i}$ are approximated through 2-exact reconstruction polynomials, the flux integral can be estimated through

$$
\iint_{A_{\alpha \beta}}(\mathbf{u} \phi) \cdot \mathbf{n} \mathrm{d} A=\dot{m}_{\Gamma} \phi_{\Gamma}+\left.\dot{m}_{i, \Gamma} \frac{\partial \phi}{\partial x_{i}}\right|_{\mathbf{x}_{\Gamma}}+\left.\frac{1}{2} \dot{m}_{i j, \Gamma} \frac{\partial^{2} \phi}{\partial x_{i} \partial x_{j}}\right|_{\mathbf{x}_{\Gamma}}+\mathcal{O}\left(h^{3}\right) .
$$

The terms $\dot{m}_{\Gamma}, \dot{m}_{j, \Gamma}$ and $\dot{m}_{k l, \Gamma}$, referred to as massflux tensors of rank zero, one and two and can be calculated according to

$$
\begin{aligned}
\dot{m}_{\Gamma} & :=u_{i, \Gamma} \mathcal{S}_{i}^{(\alpha \beta)}+\left.\frac{\partial u_{i}}{\partial x_{j}}\right|_{\mathbf{x}_{\Gamma}} \mathcal{S}_{i, j}^{(\alpha \beta)}+\left.\frac{1}{2} \frac{\partial^{2} u_{i}}{\partial x_{j} \partial x_{k}}\right|_{\mathbf{x}_{\Gamma}} \mathcal{S}_{i, j k}^{(\alpha \beta)}, \\
\dot{m}_{i, \Gamma} & :=u_{j, \Gamma} \mathcal{S}_{j, i}^{(\alpha \beta)}+\left.\frac{\partial u_{j}}{\partial x_{k}}\right|_{\mathbf{x}_{\Gamma}} \mathcal{S}_{j, k i}^{(\alpha \beta)} \\
\dot{m}_{i j, \Gamma} & :=u_{k, \Gamma} \mathcal{S}_{k, i j}^{(\alpha \beta)}
\end{aligned}
$$

The terms $\mathcal{S}^{(\alpha \beta)}$ are designated as rank $p$ geometric surface tensors and arise due to the surface-integration of the Taylor-series expansion. They are defined by

$$
\mathcal{S}_{i, j_{1} j_{2} \ldots j_{p}}^{(\alpha \beta)}:=\iint_{A_{\alpha \beta}} n_{i}\left(x_{j_{1}}-x_{j_{1}, \Gamma}\right)\left(x_{j_{2}}-x_{j_{2}, \Gamma}\right) \ldots\left(x_{j_{p}}-x_{j_{p}, \Gamma}\right) \mathrm{d} A .
$$

The subscripts $i$ and $j_{p}$ are separated by a comma, in order to highlight that $i$ indicates the face normal direction and $j_{p}$ the spatial direction of the distance terms $\left(x_{j_{p}}-x_{j_{p}, \Gamma}\right)$. The superscript in brackets specifies 
the adjacent elements $\Omega_{\alpha}$ and $\Omega_{\beta}$ of the face on which point $\mathbf{x}_{\Gamma}$ for the Taylor-series expansion is located. The rank 0 geometric surface tensor $\mathcal{S}_{i}^{(\alpha \beta)}$ denotes the joint normal of all sub-faces of a median dual cell face $A_{\alpha \beta}$. All surface tensors depend solely on the geometry of the median-dual grid and can be computed in a preprocessing step prior to the simulation. The massflux tensors at the interface node $\mathbf{x}_{\Gamma}$ are calculated with a central averaging of the reconstructed values from both adjacent elements. The scalar field variable $\phi_{\Gamma}$ is approximated according to

$$
\begin{aligned}
\phi_{\Gamma} & =\alpha_{U} \phi_{U}+\alpha_{D} \phi_{D} \\
& +\left(\beta_{U} \frac{\partial}{\partial x_{i}}[\bar{\phi}]_{U}^{* *}+\beta_{D} \frac{\partial}{\partial x_{i}}[\bar{\phi}]_{D}^{* *}\right) \Delta x_{i} \\
& +\left(\gamma_{U} \frac{\partial^{2}}{\partial x_{i} \partial x_{j}}[\bar{\phi}]_{U}^{*}+\gamma_{D} \frac{\partial^{2}}{\partial x_{i} \partial x_{j}}[\bar{\phi}]_{D}^{*}\right) \Delta x_{i} \Delta x_{j},
\end{aligned}
$$

where $U$ is the upwind and $D$ the downwind direction at the interface and $\Delta x_{i}=x_{i, D}-x_{i, U}$ denotes the distance vector from the upwind to the downwind node. It must be noted, that $\phi_{U}$ and $\phi_{D}$ are values of $\phi$ at the respective primary grid nodes and must be approximated from the underlying reconstruction polynomial according to equation (3). The derivatives of $\phi$ at the interface are reconstructed analogously through

$$
\begin{gathered}
\left.\frac{\partial \phi}{\partial x_{i}}\right|_{\mathbf{x}_{\Gamma}}=\alpha_{U} \frac{\partial}{\partial x_{i}}[\bar{\phi}]_{U}^{* *}+\alpha_{D} \frac{\partial}{\partial x_{i}}[\bar{\phi}]_{D}^{* *}+\left(\beta_{U} \frac{\partial^{2}}{\partial x_{i} \partial x_{j}}[\bar{\phi}]_{U}^{*}+\beta_{D} \frac{\partial^{2}}{\partial x_{i} \partial x_{j}}[\bar{\phi}]_{D}^{*}\right) \Delta x_{j}, \\
\left.\frac{\partial^{2} \phi}{\partial x_{i} \partial x_{j}}\right|_{\mathbf{x}_{\Gamma}}=\alpha_{U} \frac{\partial^{2}}{\partial x_{i} \partial x_{j}}[\bar{\phi}]_{U}^{*}+\alpha_{D} \frac{\partial^{2}}{\partial x_{i} \partial x_{j}}[\bar{\phi}]_{D}^{*} .
\end{gathered}
$$

By adjustment of the coefficients $\alpha, \beta, \gamma$ it is possible to blend between different discretization schemes. Table 1 shows parameters that lead to a 2 -exact, a 1 -exact and to a 0 -exact, conventional central differencing scheme (CDS). Depending on the exactness of the scheme, the respective correction operations on the derivatives are applied. For the 0-exact schemes, point values such as $\phi_{U}$ reduce to volume averages $\bar{\phi}_{U}$. The parameter $\theta$ is utilized for the 2- and 1-exact schemes in order to apply a bias towards the element of the upwind direction and thus stabilizing the solution.

Table 1: Parameters for the flux calculation with different discretization schemes.

\begin{tabular}{lcccccc} 
Scheme & $\alpha_{U}$ & $\alpha_{D}$ & $\beta_{U}$ & $\beta_{D}$ & $\gamma_{U}$ & $\gamma_{D}$ \\
\hline$k=2$ & $(1+\theta) / 2$ & $(1-\theta) / 2$ & $(1+\theta) / 4$ & $-(1-\theta) / 4$ & $(1+\theta) / 8$ & $(1-\theta) / 8$ \\
$k=1$ & $(1+\theta) / 2$ & $(1-\theta) / 2$ & $(1+\theta) / 4$ & $-(1-\theta) / 4$ & 0 & 0 \\
CDS & $1 / 2$ & $1 / 2$ & 0 & 0 & 0 & 0
\end{tabular}

\section{Analysis of $k$-Exact Correction Matrices}

The eigenvalues of the matrices $\mathbf{G}_{\alpha}$ and $\mathbf{H}_{\alpha}$ are analyzed for $d=3$ with a primary grid featuring $6 \times 6 \times 6$ hexahedral elements of equal size. The matrices are calculated for the median dual grid element $\Omega_{\alpha}$, which is located at the center of the entire domain and whose surface is defined by 27 vertices. To apply a deformation on the element, its primary grid node $\mathbf{x}_{\alpha}$ is displaced by an amount of $\delta x$ in any direction in space, prior to the construction of the median dual elements. The displacement results in a deformation of the respective element and also its direct neighbors, as already shown for $d=2$ in Figure 2 For the further analysis, the displacement $\delta x$ is adjusted in three different directions, which are shown in Figure 3 To get an overview on how the element is distorted with respect to the displacement of $\mathbf{x}_{\alpha}$, the aspect ratio $\mathcal{A}_{\alpha}$ is calculated for the median dual element $\Omega_{\alpha}$ according to the following definition:

$$
\mathcal{A}_{\alpha}=\frac{\max \left(\left\|\mathcal{S}^{\alpha \beta}\right\|\right)}{\min \left(\left\|\mathcal{S}^{\alpha \beta}\right\|\right)}, \quad \forall \beta \in \mathbb{V}_{\alpha}^{(1)},
$$

with the surface area $\left\|\mathcal{S}^{\alpha \beta}\right\|$. The variation of the aspect ratio for $\Omega_{\alpha}$ is shown in Figure $4 \mathrm{a}$. The maximum displacements $\delta x$ vary with the direction, in order to keep the primary grid node $\mathbf{x}_{\alpha}$ contained within the 


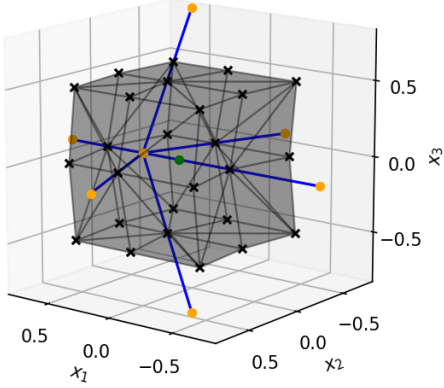

(a) Displacement in $x_{1}$-direction

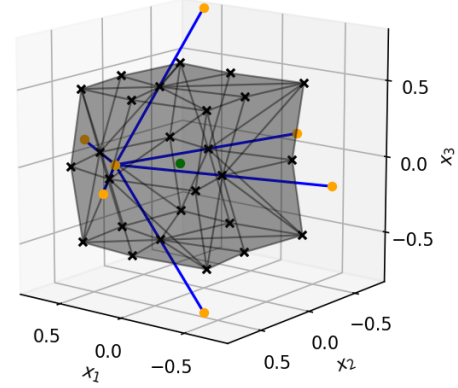

(b) Displacement in $x_{1}-x_{2}$-direction

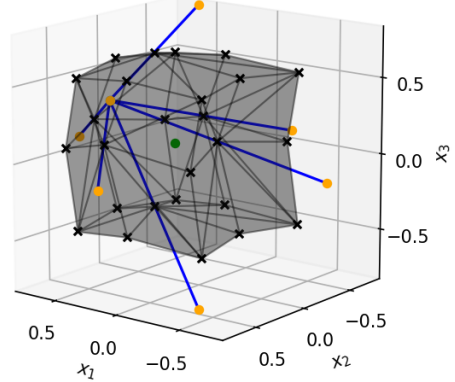

(c) Displacement in $x_{1}-x_{2}-x_{3}$-direction

Figure 3: Directions of displacement used for the investigation of the matrix eigenvalues. Yellow dots represent primary grid nodes, the green dot represents the geometric center of the median dual element.

median dual element. Generally, the aspect ratio rises with the displacement of the pirmary grid node $\mathbf{x}_{\alpha}$. Only slight variations are obtained, when the direction of $\delta x$ is changed. The lowest values are obtained, when a displacement in the $x_{1}-x_{2}-x_{3}$ direction is applied. In contrast to the aspect ratio, Figures $4 \mathrm{~b}$ and $4 \mathrm{c}$ show how the minimum and maximum eigenvalues of the gradient correction matrix $\mathbf{G}_{\alpha}$ change with the displacement $\delta x$. For a non-distorted element with $\delta x=0$, all eigenvalues equal to a value of one. With increasing displacement, the minimum eigenvalues fall below a value of one, whereas the maximum eigenvalues increase. If a unidirectional displacement in $x_{1}$-direction is applied, the minimum eigenvalue decrease is stronger than for the displacement in $x_{1}-x_{2}$ direction. The lowest decrease appears for the direction $x_{1}$ $x_{2}-x_{3}$. Similarly, the maximum eigenvalues incerease to a greater extent for both $x_{1}$ - and $x_{1}-x_{2}$-direction. Interestingly, all eigenvalues of $\mathbf{G}_{\alpha}$ consist only of real parts, independent of the amount or the direction of $\delta x$. A different behaviour is observed for the eigenvalues of the Hessian correction matrix $\mathbf{H}_{\alpha}$, which also feature complex parts in certain areas of the displacement space. The locations of $\mathbf{x}_{\alpha}$ at which $\mathbf{H}_{\alpha}$ contains complex eigenvalues are indicated as red dots in Figure 5a. The blue dots are the locations, at which the eigenvalues of $\mathbf{H}_{\alpha}$ only contain real parts. It seems that complex eigenvalues occur at positions symmetric to the geometric centroid of the non-distorted element. Since matrix $\mathbf{G}_{\alpha}$ only contains real parted eigenvalues, the correction operation in equation (8) can be seen as a scaling of the 0-exact gradient operator, such that

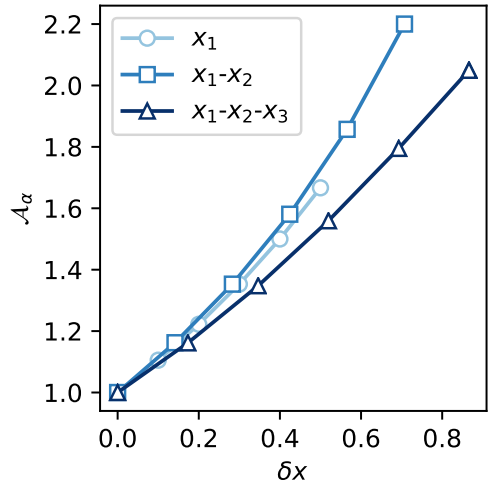

(a) Aspect ratio $\mathcal{A}_{\alpha}$

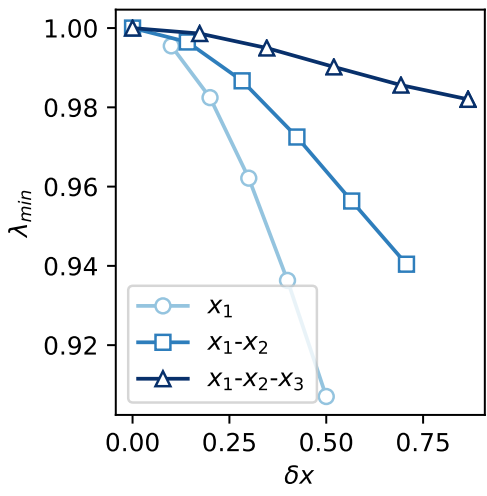

(b) Minimum eigenvalue $\lambda_{\min }$

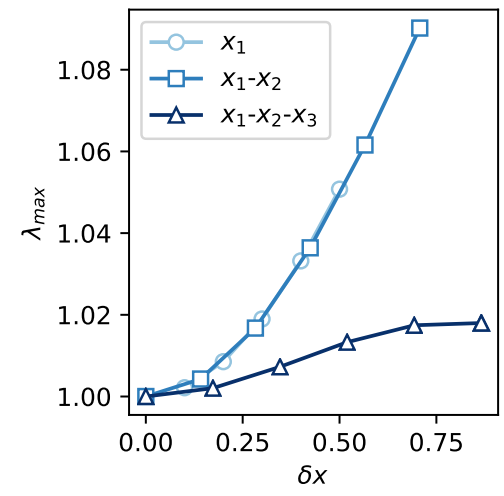

(c) Maximum eigenvalue $\lambda_{\max }$

Figure 4: Variation of the element aspect ratio and the eigenvalues of the gradient correction matrix $\mathbf{G}_{\alpha}$ with displacement $\delta x$ of the primary grid node $\mathbf{x}_{\alpha}$ into various directions. 


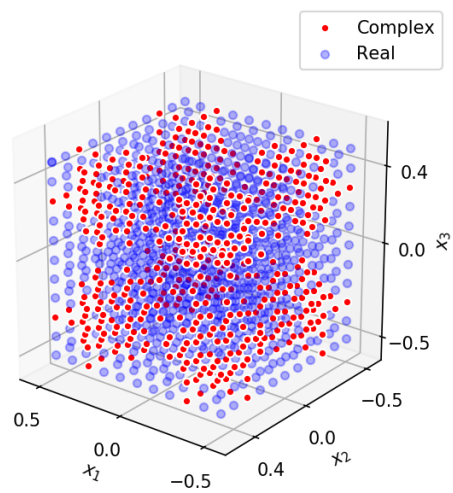

(a) Appearance of real and complex eigenvalues. Dots highlight the primary grid node location $\mathbf{x}_{\alpha}$

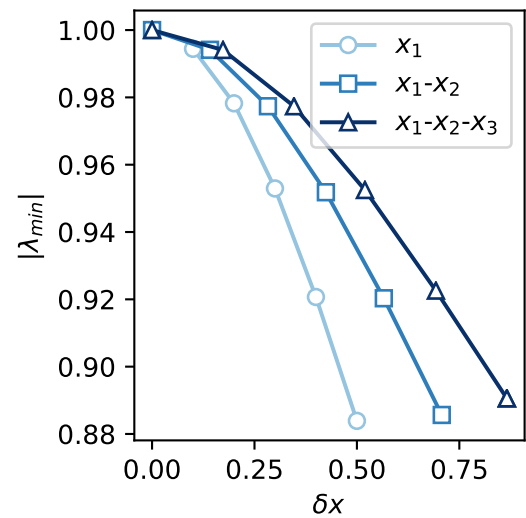

(b) Minimum absolute eigenvalue $\left|\lambda_{\min }\right|$

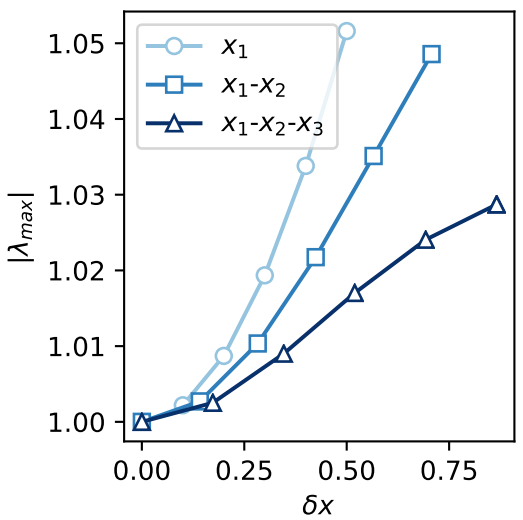

(c) Maximum absolute eigenvalue $\left|\lambda_{\max }\right|$

Figure 5: Variation of the eigenvalues of the Hessian correction matrix $\mathbf{G}_{\alpha}$ with displacement $\delta x$ of the primary grid node $\mathbf{x}_{\alpha}$ into various directions.

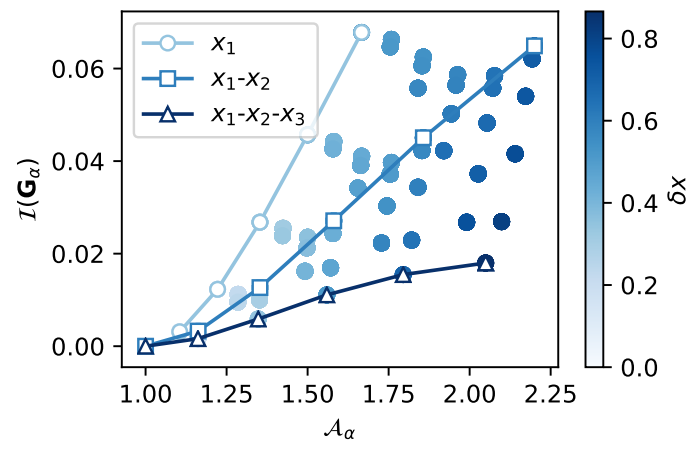

(a) Indicator calculated from eigenvalues of $\mathbf{G}_{\alpha}$

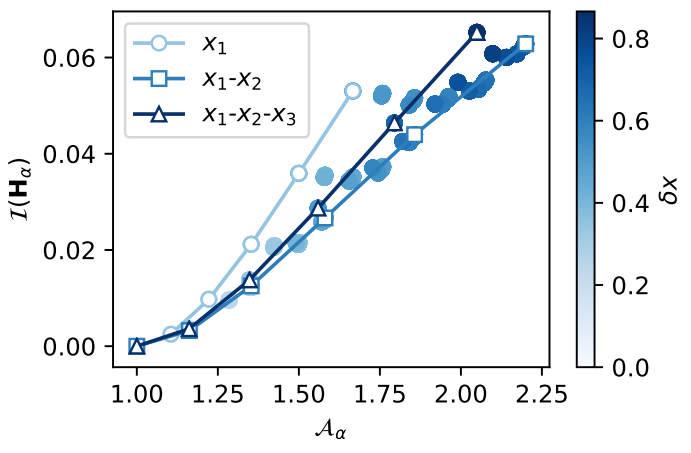

(b) Indicator calculated from eigenvalues of $\mathbf{H}_{\alpha}$

Figure 6: Variation of the indicator $\mathcal{I}$ and the aspect ratio $\mathcal{A}_{\alpha}$ with primary grid node displacement $\delta x$.

it satisfies the constraints for a 1-exact reconstruction polynomial, which is essentially a linear function. Contrary, matrix $\mathbf{H}_{\alpha}$ is needed for the correction of a 2-exact polynomial, which introduces a further degree of freedom. The appearance of complex eigenvalues can thus be interpreted as a rotatory operation applied on the 0-exact Hessian matrix operator in Equation (13). The absolute minmum and maximum eigenvalues of the Hessian correction matrix change in a similar manner with the displacement as for matrix $\mathbf{G}_{\alpha}$. This is shown in Figures 5b and 5c. Similarly to the gradient correction matrix, all eigenvalues of $\mathbf{H}_{\alpha}$ equal to a value of one for the undistorted element.

In order to incorporate all eigenvalues into a single metric, we introduce the indicator $\mathcal{I}$. It is calculated through the $L_{2}$-norm of the eigenvalues for matrix $\mathbf{G}_{\alpha}$ and $\mathbf{H}_{\alpha}$ respectively

$$
\mathcal{I}=\sqrt{\frac{1}{N_{\lambda}} \sum_{i=1}^{N_{\lambda}}\left(\lambda_{i}-1\right)^{2}} .
$$

Figure 6 shows the correlation of $\mathcal{I}$ with the aspect ratio $\mathbf{A}_{\alpha}$ for both gradient and Hessian correction matrices. The points refer to different displacements $\delta x$ and the three lines denote the paths of displacement along a specified direction. For both matrices, the indicator $\mathcal{I}$ equals to zero if no distortion is applied. The

$$
8 \text { of } 13
$$


high variation of $\mathcal{I}\left(\mathbf{G}_{\alpha}\right)$ over the aspect ratio indicates, that the introduced grid metric incorporates a higher amount of information on the distortion level of the grid. This behaviour is less distinct for matrix $\mathbf{H}_{\alpha}$, since both the displacement in $x_{1}-x_{2}$ and $x_{1}-x_{2}-x_{3}$ direction produce similar values. Hence the main focus of the following work will be on the inidcator $\mathcal{I}\left(\mathbf{G}_{\alpha}\right)$.

\section{Application to Linear Convection Test Case}

The indicator $\mathcal{I}\left(\mathbf{G}_{\alpha}\right)$ is furthermore investigated through the solution of equation (3) on different grids and for the discretization schemes proposed in Table 1 . A value of $\theta=0$ is employed for the $k$-exact schemes, since no upwinding is required to stabilize the solution. We consider the convection of a scalar quantity $\phi$, whose initial distribution is a Gaussian pulse function

$$
\phi(\mathbf{x}, t=0)=\beta U_{\infty} e^{-\frac{\left(x-x_{0}\right)^{2}+\left(y-y_{0}\right)^{2}}{\sigma^{2}}},
$$

with $\mathbf{x}_{0}=[L / 2, L / 2]^{T}$. The scalar is transported in a uniform flow field $\mathbf{u}=\left[U_{\infty}, 0\right]^{T}$ through a domain $\mathbf{x} \in[0, L]^{2}$ with unit length $L$. Table 2 shows the employed parameters for the test case. Periodic boundary conditions are utilized in $x_{1}$-direction, whereas for the $x_{2}$-direction symmetry boundaries are applied. A convection distance of $3 L$ is used and the degrees of freedom are set to $N_{\text {DOF }}=\left\{16^{2}, 32^{2}, 64^{2}, 128^{2}, 256^{2}\right\}$. The Crank-Nicolson scheme is utilized for the temporal discretization and a CFL number of 0.1 is employed for all simulations, in order to reduce the influence of temporal discretization errors

$$
\frac{\phi^{n+1}-\phi^{n}}{\Delta t}+\frac{1}{2} \nabla \cdot(\mathbf{u} \phi)^{n+1}+\frac{1}{2} \nabla \cdot(\mathbf{u} \phi)^{n}=0 .
$$

Table 2: Parameters used for the linear advection test case.

\begin{tabular}{lcccc} 
Parameter & $L$ & $U_{\infty}$ & $\beta$ & $\sigma$ \\
\hline Value & 0.1 & 20 & 0.02 & 0.0075
\end{tabular}

Three different grid types with different levels of distortion are employed, which are shown in Figure 7. The first two primary grid types consists only of triangles, whereas the third grid also incorporates quadrilateral elements. For this arrangement of elements, the median dual elements feature a lower number of direct neighbors and the connectivity-direction is arranged more anisotropicly. As it will be shown below, this anisotropy significantly reduces the accuracy of the conventional $C D S$ scheme, even though the number of degrees of freedom remains the same and no element distortion is applied. Each grid can be characterized by means of a histogram of estimated indicator values and the respective ensemble means $\overline{\mathcal{I}\left(\mathbf{G}_{\alpha}\right)}$, as shown in Figures 8 to 10. For reasons of clarity, the histograms for the coarse grid with $N_{\text {DOF }}=16^{2}$ is not shown.

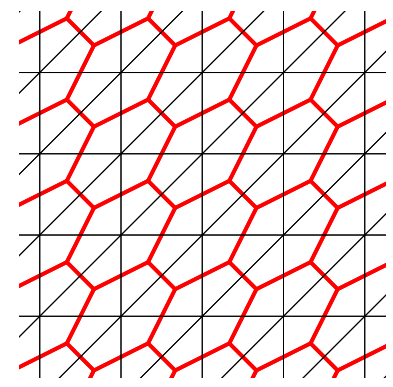

(a) Right triangles

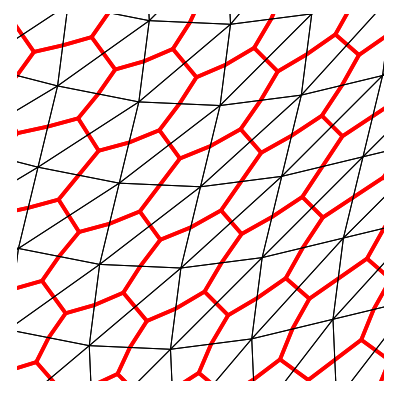

(b) Skewed triangles

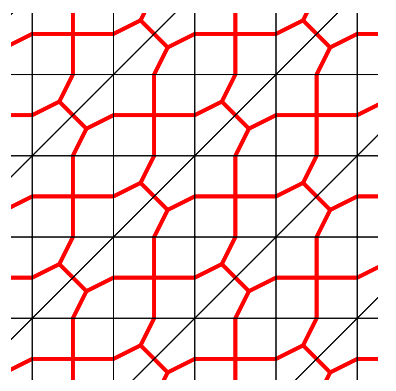

(c) Right triangles and quadrilaterals

Figure 7: Grids used for the linear convection test case. Primary grid $\mathcal{P}(\Omega)$ connectivities are shown in black and the respective median dual grid representation $\mathcal{D}(\Omega)$ in red. 
The grids with right triangles feature the lowest mean values with a distinct peak in the histograms near a value of zero, as shown in Figures $8 \mathrm{a}$ to $8 \mathrm{~d}$. The small peaks at $\mathcal{I}\left(\mathbf{G}_{\alpha}\right) \approx 0.1$ are artifacts due to the applied symmetry boundary conditions in $x_{2}$-direction, where adjacent elements feature different geometric moment tensors. These peaks diminish as the number of degrees of freedom is rising, since the overall share of boundary elements is decreasing. This leads also to the fact, that the mean indicator value decreases with higher values of $N_{\text {DOF }}$. The meshes with deformed triangles feature broader distributions of $\mathcal{I}\left(\mathbf{G}_{\alpha}\right)$ and higher mean values than the right triangle mesh. However, as the domain is discretized by a sufficient number of elements, the effect of deformation becomes negligible which is visible through the sharp histogram profile for $N_{\mathrm{DOF}}=256^{2}$ in Figure 9d. The histograms in Figures 10 are calculated for the meshes with mixed elements and show the highest values for $\mathcal{I}\left(\mathbf{G}_{\alpha}\right)$, which are present by means of two dominant peaks. These peaks arise regardless of $N_{\mathrm{DOF}}$ and can be associated with the two primary grid element types. The mean indicator values differ by about an order of magnitude compared to the other grid types. Interestingly, this occurs even though the employed primary grid elements are not deformed at all. The fact, that these two distinct peaks do not disappear as $N_{\text {DOF }}$ is increased supports the hypothesis that the indicator $\mathcal{I}\left(\mathbf{G}_{\alpha}\right)$ reflects the anisotropic connectivity of the median dual elements of this grid type.

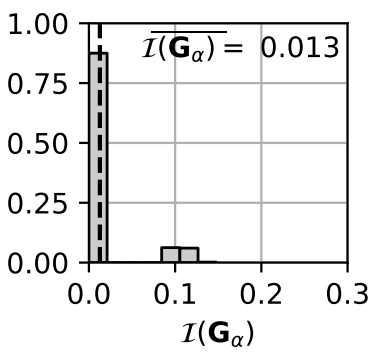

(a) $N_{\mathrm{DOF}}=32^{2}$

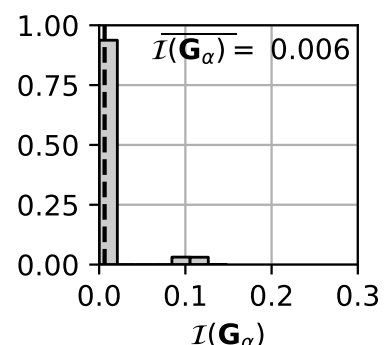

(b) $N_{\mathrm{DOF}}=64^{2}$

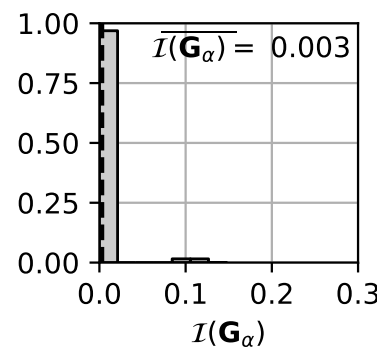

(c) $N_{\mathrm{DOF}}=128^{2}$

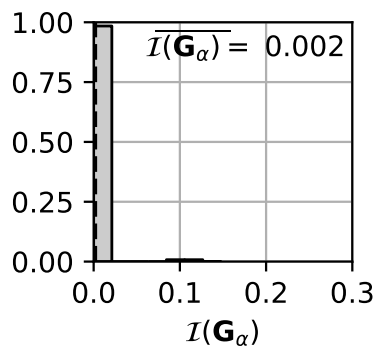

(d) $N_{\mathrm{DOF}}=256^{2}$

Figure 8: Histograms of indicator $\mathcal{I}\left(\mathbf{G}_{\alpha}\right)$ for grids consisting of right triangles.

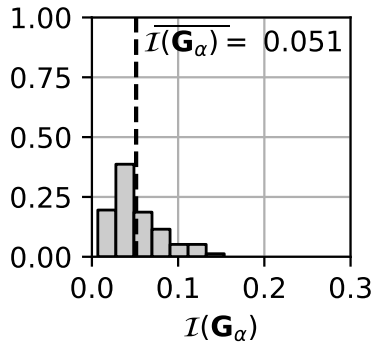

(a) $N_{\text {DOF }}=32^{2}$

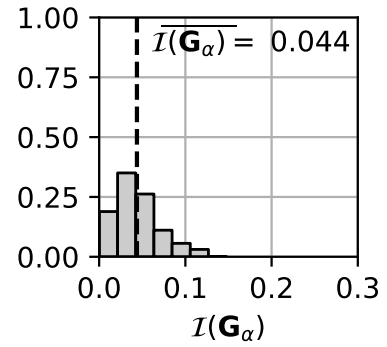

(b) $N_{\mathrm{DOF}}=64^{2}$

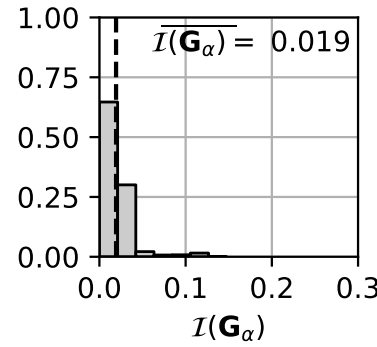

(c) $N_{\mathrm{DOF}}=128^{2}$

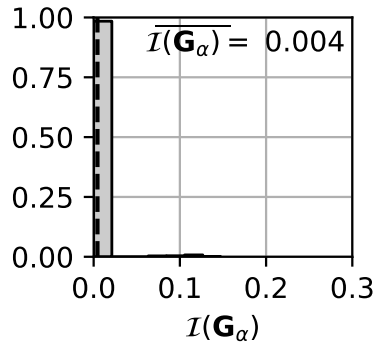

(d) $N_{\mathrm{DOF}}=256^{2}$

Figure 9: Histograms of indicator $\mathcal{I}\left(\mathbf{G}_{\alpha}\right)$ for grids consisting of skewed triangles.

Figure 11 shows results of the convergence study of the linear convection test case for various grids and discretization schemes. The numerical error is expressed by means of the $L_{2}$-norm $E_{L_{2}(\Omega)}$

$$
E_{L_{2}(\Omega)}=\left[\frac{\sum_{\alpha=1}^{N_{\mathrm{DOF}}}\left(\bar{\phi}_{\alpha}-\bar{\phi}_{\alpha}^{\mathrm{ex}}\right)^{2}\left|\Omega_{\alpha}\right|}{\sum_{\alpha=1}^{N_{\mathrm{DOF}}}\left|\Omega_{\alpha}\right|}\right]^{1 / 2},
$$

with the volume-averaged, exact solution $\bar{\phi}_{\alpha}^{\mathrm{ex}}$ and the total number of elements $N_{\text {DOF }}$. For both the grids that consist of right and skewed triangles, all three schemes preserve their prescribed levels of accuracy as the grids 


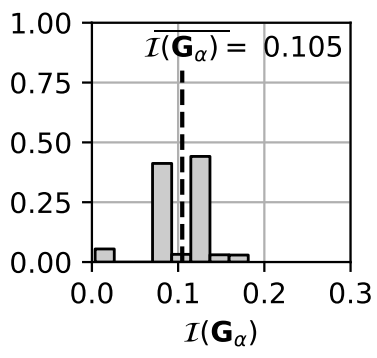

(a) $N_{\mathrm{DOF}}=32^{2}$

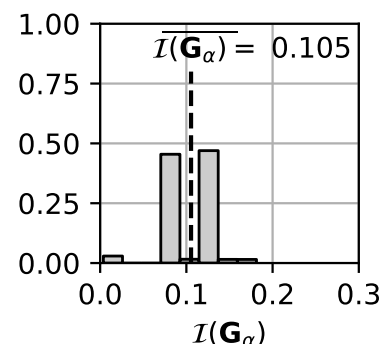

(b) $N_{\mathrm{DOF}}=64^{2}$

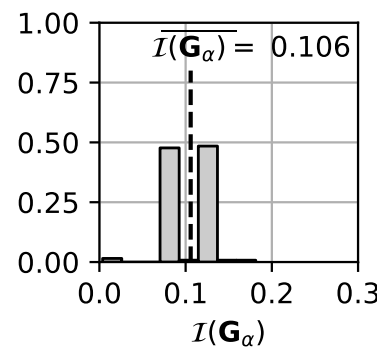

(c) $N_{\mathrm{DOF}}=128^{2}$

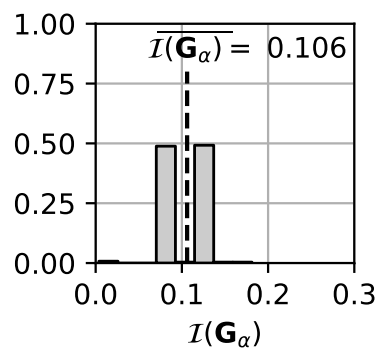

(d) $N_{\mathrm{DOF}}=256^{2}$

Figure 10: Histograms of indicator $\mathcal{I}\left(\mathbf{G}_{\alpha}\right)$ for grids consisting of right triangles and quadrilaterals.
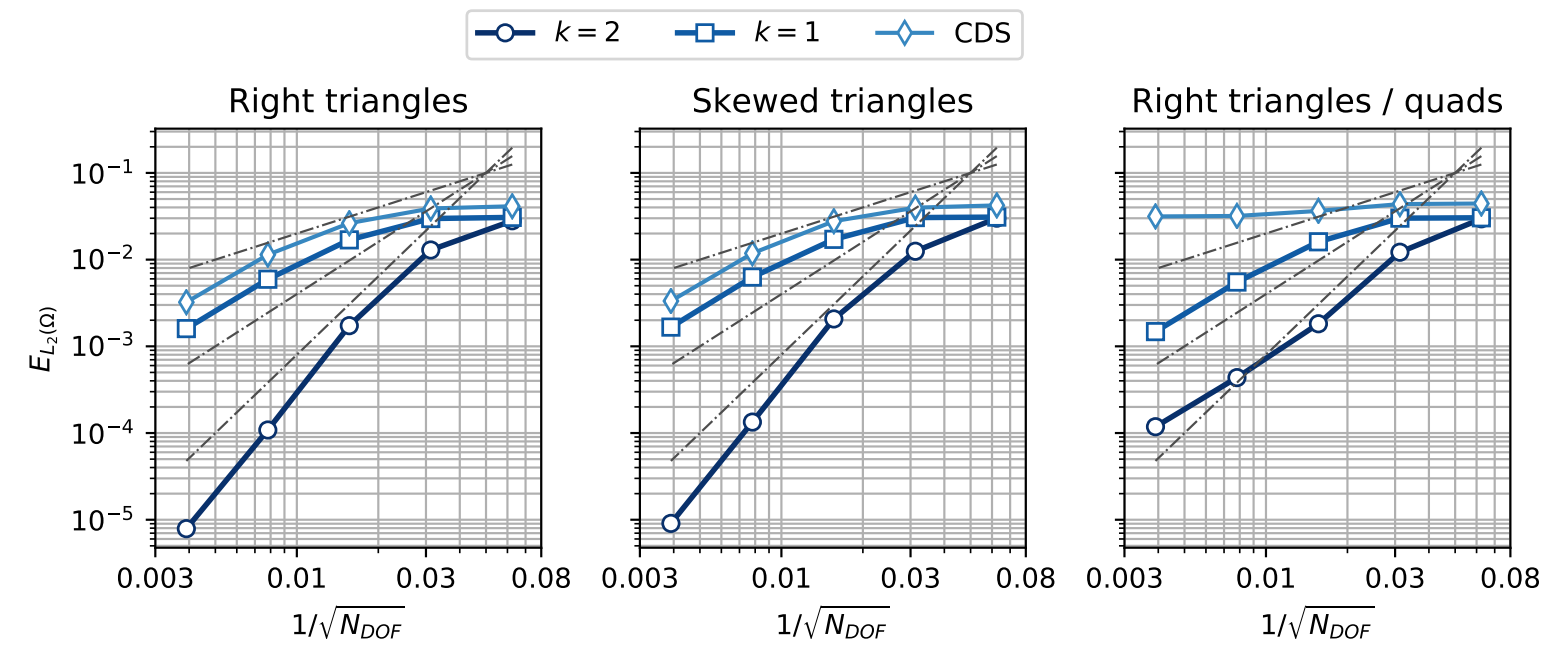

Figure 11: Error convergence for the linear advection test case with varying degrees of freedom on different grids. The dashed lines represent error slopes $\mathcal{O}\left(h^{3}\right), \mathcal{O}\left(h^{2}\right)$ and $\mathcal{O}(h)$. The exactness of the reconstruction poylnomial is given by the value of $k$.

are refined. The 2-exact scheme features the lowest errors, followeder by the 1-exact reconstruction method and the conventional $C D S$ scheme. Comparing the results from both grids shows that the deformation of the triangular elements results in a shift of the convergence curves towards higher error levels. This error is relatively small for all schemes and is majorly noticeable for the 2-exact scheme. A different result is obtained, when the mesh is employed that consists of both quadrilateral and triangular grid elements, as shown on the right in Figure 11. Regardless of the grid resolution, the $C D S$ scheme leads to an invalid solution for this grid type. This causes the respective error curve to remain constant even when the finest grid resolution is used. In contrast, both 1- and 2-exact methods preserve the solution with nearly the same accuracies as for the right triangle mesh. However, for $k=2$ the overall order of accuracy slightly reduces to $\mathcal{O}\left(h^{2}\right)$. The solution that is obtained with all three schemes on the mixed element grid is highlighted in Figure 12 The $k$-exact schemes both preserve the shape of the Gaussian pulse well, even though the 1-exact solution features a higher dispersive and dissipative error. In contrast to this, the shape of the pulse in the solution with the conventional $C D S$ scheme is almost no more to be recognized. It should be noted, that this results only due to the fact that this grid type offers less interconnections between the primary grid nodes than the right triangle mesh. It can also be stated, that both $k$-exact methods are generally better in handling such anisotropic meshes than the conventional discretization approach.

Figure 13 shows the relation between the numerical error and the ensemble mean indicator $\overline{\mathcal{I}\left(\mathbf{G}_{\alpha}\right)}$ calculated for the respective grids. It can be observed for both $k$-exact schemes, that the numerical error remains 


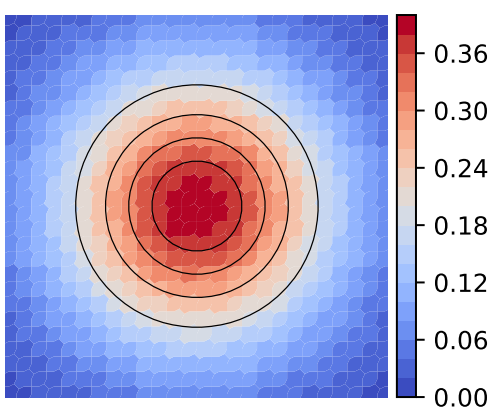

(a) $k=2$

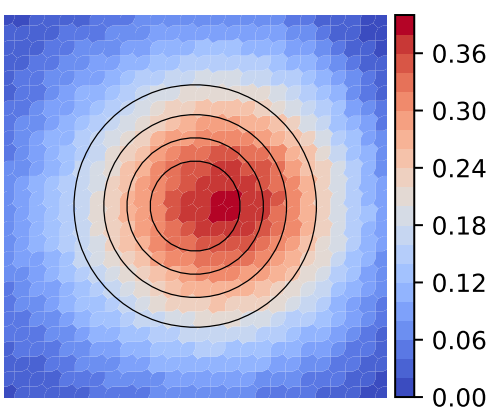

(b) $k=1$

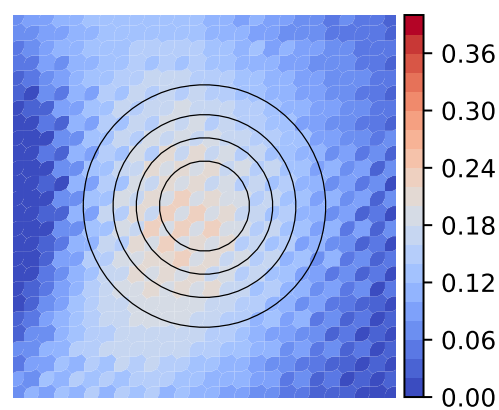

(c) CDS

Figure 12: Solution of $\bar{\phi}_{\alpha}$ with different discretization schemes on the grid of right triangles and quadrilaterals with $N_{\mathrm{DOF}}=128^{2}$. The analytic solution of $\phi$ is shown by means of black iso-contours.

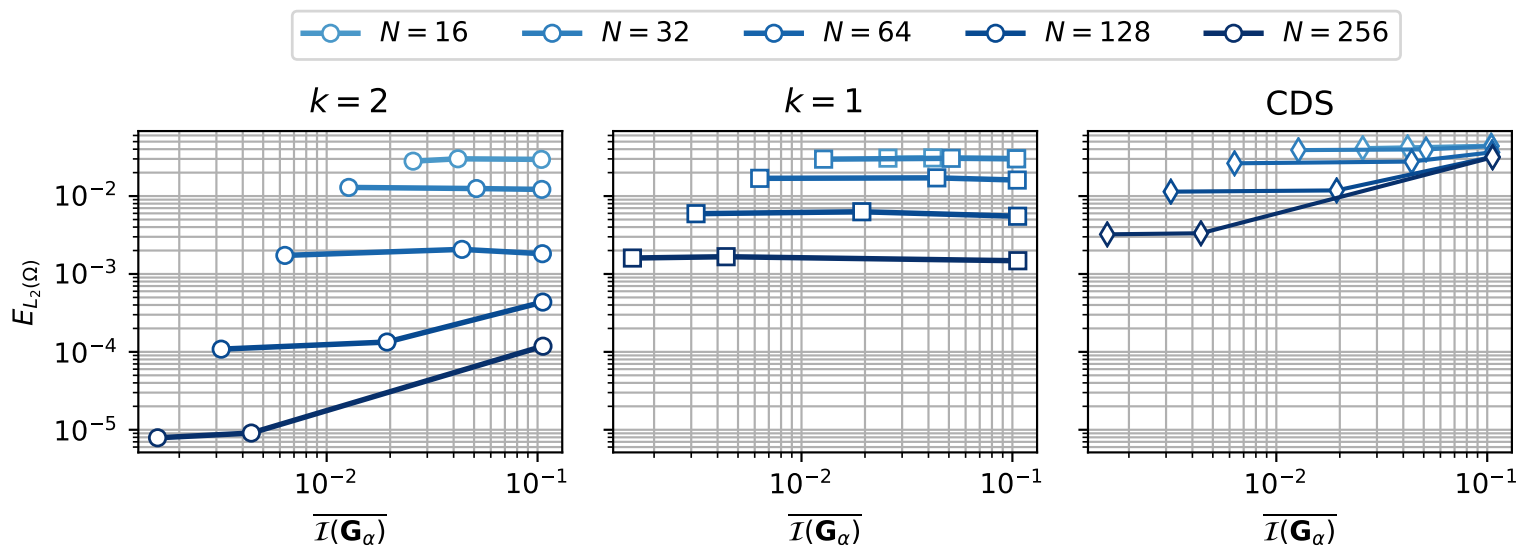

Figure 13: Error convergence of the linear advection test case with variyng indicator $\overline{\mathcal{I}\left(\mathbf{G}_{\alpha}\right)}$ for different discretization schemes. The exactness of the reconstruction poylnomial is given by the value of $k$.

constant for a desired resolution as the mean indicator values vary. However, as soon as a certain threshold is reached, the error increases with the $\overline{\mathcal{I}\left(\mathbf{G}_{\alpha}\right)}$. The threshold for the 2-exact scheme lies approximately below a value of $E_{L_{2}(\Omega)} \leq 10^{-3}$. It can not be identifiert for the 1-exact method, since the threshold has probably not been surpassed. The behaviour is similar for the conventional $C D S$ scheme, but the threshold at which the error is influenced by the mean indicator is reached for significantly higher values of $E_{L_{2}(\Omega)}$.

\section{Conclusion}

The eigenstructure of correction matrices used in the $k$-exact multiple-correction scheme for vertexcentered grids has been investigated in detail within this work. These matrices are needed for a $k$-exact correction of Green-Gauss derivatives, in order to obtain a spatially higher order of accuracy, even on deformed meshes. The impact on the eigenvalues due to deformation was shown for both the gradient and Hessian correction matrix by means of a single median dual element. It could be observed, that the eigenvalues not only reflect the amount of distortion, but also incorporate the direction of the element deformation. An indicator has been formulated based on the $L_{2}$-norm of the eigenvalues, which allows a quantitative measure for the skewness and the anisotropic connectivity of investigated grids. By employing this indicator for a linear convection test case, it was demonstrated that the order of accuracy achieved 
by the standard $C D S$ discretization scheme deteriorates with the grid anisotropy. In contrast, the $k$-exact schemes developed are quite insensitive to the grid anisotropy. Only for the finest grid resolution used a dependency can be observed for the 2-exact reconstruction, which will be investigated further. Despite this, the overall error level for the 2-exact reconstruction is the lowest of all discretization schemes compared in the paper. In summary, it could be shown that the indicator serves well as a grid metric to evaluate unstructured Finite-Volume grids. Further benchmark test cases should be investigated in future works, in order to gain a more in-depth understanding of the proposed indicator and its relation to different grades of grid distortion. It should also be examined wether the indicator can be used to enhance the element quality for adaptive-grid simulations with anisotropic mesh adaptation.

\section{References}

${ }^{1}$ Ekaterinaris, J. A., "High-Order Accurate, Low Numerical Diffusion Methods for Aerodynamics," Progress in Aerospace Sciences, Vol. 41, No. 3-4, 2005, pp. 192-300.

${ }^{2}$ Huynh, H., Wang, Z. J., and Vincent, P. E., "High-Order Methods for Computational Fluid Dynamics: A Brief Review of Compact Differential Formulations on Unstructured Grids," Computers 85 fluids, Vol. 98, 2014, pp. 209-220.

${ }^{3}$ Barth, T. and Frederickson, P., "Higher Order Solution of the Euler Equations on Unstructured Grids using Quadratic Reconstruction," 28th Aerospace Sciences Meeting, 1990, p. 13.

${ }^{4}$ Barth, T. J., "Aspects of Unstructured Grids and Finite-Volume Solvers for the Euler and Navier-Stokes Equations," 31st Aerospace Sciences Meeting, 1992, pp. 668.

${ }^{5}$ Haider, F., Bertier, N., Courbet, B., Vuillot, F., and Croisille, J., "Application of a High Order Finite Volume Scheme on Unstructured Grids to Fluid Dynamics and Aerothermochemistry," hal-01111360.

${ }^{6}$ Haider, F., Brenner, P., Courbet, B., and Croisille, J. P., "Efficient Implementation of High Order Reconstruction in Finite Volume Methods," Finite Volumes for Complex Applications VI - Problems and Perspectives, Springer Proceedings in Mathematics, Vol. 4, 2011, pp. 553-560.

${ }^{7}$ Haider, F., Discrétisation en maillage non structuré général et applications LES, Ph.D. thesis, Université Pierre et Marie Curie-Paris VI, 2009.

${ }^{8}$ Pont, G., Brenner, P., Cinnella, P., Maugars, B., and Robinet, J.-C., "Multiple-correction hybrid k-exact schemes for high-order compressible RANS-LES simulations on fully unstructured grids," Journal of Computational Physics, Vol. 350, 2017, pp. $45-83$.

${ }^{9}$ Setzwein, F., Ess, P., and Gerlinger, P., "High-Order k-Exact Finite Volume Scheme for Vertex-Centered Unstructured Grids," AIAA Scitech 2020 Forum, 2020, p. 1785.

${ }^{10}$ Ollivier-Gooch, C. and Van Altena, M., "A High-Order-Accurate Unstructured Mesh Finite-Volume Scheme for the Advection-Diffusion Equation," Journal of Computational Physics, Vol. 181, No. 2, 2002, pp. 729-752.

${ }^{11}$ Charest, M. R., Canfield, T. R., Morgan, N. R., Waltz, J., and Wohlbier, J. G., "A High-Order Vertex-Based Central ENO Finite-Volume Scheme for Three-Dimensional Compressible Flows," Computers \& Fluids, Vol. 114, 2015, pp. 172-192. 